\title{
Comparison of migration disturbance potency of epigallocatechin gallate (EGCG) synthetic analogs and EGCG PEGylated PLGA nanoparticles in rat neurospheres.
}

\author{
Britta A. Kühne ${ }^{a}$, Teresa Puig ${ }^{b}$, Santiago Ruiz-Martínez ${ }^{b}$, Joan Crous-Masób,c, Marta Planas ${ }^{c}$, \\ Lidia Feliuc ${ }^{c}$, Amanda Cano ${ }^{\text {d,e,f }}$, Maria Luisa Garcia ${ }^{\mathrm{e}, \mathrm{f}}$, Ellen Fritsche ${ }^{\mathrm{g}}$, Joan M Llobet ${ }^{\mathrm{a}, \mathrm{h}}$, Jesús \\ Gómez-Catalán ${ }^{\mathrm{a}, \mathrm{h} *}$, Marta Barenys ${ }^{\mathrm{a}, \mathrm{h} * *}$. \\ a Department of Pharmacology, Toxicology and Therapeutic Chemistry, Faculty of Pharmacy and \\ Food Sciences, University of Barcelona, 08028 Barcelona, Spain. \\ ${ }^{\mathrm{b}}$ New Therapeutic Targets Laboratory (TargetsLab), Department of Medical Sciences, University of \\ Girona, Girona Institute for Biomedical Research, 17003 Girona, Spain. \\ c LIPPSO, Department of Chemistry, University of Girona, 17003 Girona, Spain. \\ d Department of Pharmacy, Pharmaceutical Technology and Physical Chemistry, Faculty of Pharmacy \\ and Food Sciences, University of Barcelona, 08028 Barcelona, Spain. \\ e Institute of Nanoscience and Nanotechnology (IN2UB), 08028 Barcelona, Spain. \\ ${ }^{f}$ Biomedical Research Networking Centre in Neurodegenerative Diseases (CIBERNED), 28031 Madrid, \\ Spain. \\ gIUF - Leibniz Research Institute for Environmental Medicine, 40225 Düsseldorf, Germany. \\ ${ }^{h}$ Institut de Recerca en Nutrició i Seguretat Alimentària de la UB (INSA.UB), 08921 Santa Coloma de \\ Gramenet, Spain.
}

*Correspondence: jesusgomez@ub.edu ; Department of Pharmacology, Toxicology and Therapeutic Chemistry, Faculty of Pharmacy and Food Sciences, University of Barcelona, 08028 Barcelona, Spain.

**Correspondence: mbarenys@ub.edu ; Department of Pharmacology, Toxicology and Therapeutic Chemistry, Faculty of Pharmacy and Food Sciences, University of Barcelona, 08028 Barcelona, Spain.

\begin{abstract}
Epigallocatechin gallate (EGCG), the main catechin of green tea, is described to have potential health benefits in several fields like oncology, neurology or cardiology. Currently, it is also under pre-clinical investigation as a potential therapeutic or preventive treatment during pregnancy against developmental adverse effects induced by toxic substances. However, the safety of EGCG during pregnancy is unclear due to its proven adverse effects on neural progenitor cells' (NPCs) migration. As lately several strategies have arisen to generate new therapeutic agents derived from EGCG, we have used the rat 'Neurosphere Assay' to characterize and compare the effects of EGCG structurally related compounds and EGCG PEGylated PLGA nanoparticles on a neurodevelopmental key event: NPCs migration. Compounds structurally-related to EGCG induce the same pattern of NPCs migration alterations (decreased migration distance, decreased formation of migration corona, chaotic orientation of cellular processes and decreased migration of neurons at higher concentrations). The potency of the compounds does not depend on the number of galloyl groups, and small structure variations can imply large potency differences. Due to their lower toxicity observed in vitro in NPCs, 4,4'-bis[(3,4,5trihydroxybenzoyl)oxy]-1,1'-biphenyl and EGCG PEGylated PLGA nanoparticles are suggested as potential future therapeutic or preventive alternatives to EGCG during prenatal period.
\end{abstract}

Keywords: Food supplements · Neural progenitor cells · Migration · Neurospheres · Developmental neurotoxicity 
Abbreviations: CEEA: Ethic Committee for Animal Experimentation; CMINC: circular migration inhibition of neural crest cells; CTB: cellTiter-Blue Reagent; DMEM: Dulbecco's modified Eagle médium; DMSO: dimethyl sulfoxide; DNT: developmental neurotoxicity; $\mathrm{EC}_{50}$ : $50 \%$ effective concentrations; EE: encapsulation efficiency; EGC: epigallocatechin; EGCG: epigallocatechin gallate; EGF: epidermal growth factor; FASN: Fatty Acid Synthase; FGF: fibroblast growth factor; G37: 1,4-bis[(3,4,5-trihydroxybenzoyl)oxy]naphthalene; $\quad$ G56: $\quad 4,4^{\prime}$-bis[(3,4,5trihydroxybenzoyl)oxy]-1,1'-biphenyl; M1: 4-hydroxy-2-naphthyl 3,4,5-trihydroxybenzoate; M2: 3-hydroxy-1-naphthyl 3,4,5-trihydroxybenzoate; Nano-EGCG: EGCG PEGylated-PLGA nanoparticles; NPCs: neural progenitor cells; PBS: phosphate buffered saline; PDL: poly-D-lysine; PEG: polyethylene glycol; PEGylated-PLGA nanoparticles: polyethylene glicosylated poly (lacticco-glycolic acid); PFA: paraformaldehyde; PI: polydispersity index; PLGA: poly (lactic-co-glycolic acid); PLGA-PEG: poly (lactic-co-glycolic acid)-polyethylene glicol; PND1: postnatal day 1; $Z_{a v}$ : average particle size; ZP: zeta potential.

\section{Introduction}

Efforts towards improving nutritional status of pregnant women require the involvement of a wide range of specialists, among them toxicologists, as efficacy testing of nutritional supplementation should always be accompanied of safety evaluations. Several national and multinational studies report on the intake of herbal-based supplements during pregnancy with high variation on the reported percentages (reviewed by Barenys et al., 2017b) and describe that 'health promotion' is one of the ten most commonly reported reasons among pregnant women for using herbal-based supplements (Kennedy et al., 2013). As these substances are not subject to classical risk assessment evaluations, their general toxicities might be known, but there is a lack of information on specific toxicities concerning subtle endpoints like developmental neurotoxicity (DNT) (Barenys et al., 2017b).

Epigallocatechin gallate (EGCG) is the most abundant catechin in green tea (Rothwell et al., 2013). It is present in herbal-based food supplements which can be easily purchased online, in food shops or in supermarkets without the advice of health professionals. It has been described to have several potential health benefits (Hollman et al., 1999; Singh et al., 2011; Yang et al., 2009) driven by its actions on numerous cellular targets involved in cell proliferation, adhesion, and migration ((Mineva et al., 2013; Shankar et al., 2008; Singh et al., 2011; Suzuki and Isemura, 
2001), but these cellular processes are also essential for correct brain development (Holmes and McCabe, 2001), suggesting a hazardous potential for EGCG on neurodevelopment.

In a previous study using human and rat neurospheres we have shown that EGCG inhibits adhesion and migration of neural progenitor cells (NPCs) and that at higher concentrations it also produces a reduction of the number of migrating young neurons in vitro, without affecting NPC proliferation (Barenys et al., 2017). An independent research group has recently reproduced these migration alteration effects in a different test model (cMINC) showing that EGCG also inhibits human neural crest cells migration in vitro (Nyffeler et al., 2017). Besides being a freely-available food-supplement, EGCG is also intensively studied at a preclinical level as a potential prenatal treatment to decrease developmental adverse effects induced by toxic substances, i.e. prevention of the neurodevelopmental adverse effects related to the fetal alcohol syndrome (Long et al., 2010; Tiwari et al., 2010), or adverse outcomes related with genetic diseases like cardiac hypertrophy and short-term memory deficits associated with Williams-Beuren syndrome (Ortiz-Romero et al., 2018) and reduction of craniofacial features associated with Down syndrome (McElyea et al., 2016).

Even if EGCG stimulates an elevated interest for therapies in adults or during prenatal development, it displays poor bioavailability and limited stability under physiological conditions (Krupkova et al., 2016; Puig et al., 2009; Turrado et al., 2012), therefore in the last years several strategies have arisen to generate new therapeutical agents derived from EGCG to overcome these limitations: e.g. the use of polyethylene glycosylated poly (lactic-co-glycolic-acid) (PEGylated-PLGA) nanoparticles to encapsulate EGCG or the synthesis of EGCG analogues. Here we tested such EGCG alternatives in the 'Neurosphere Assay' to assess their potency to disturb (1) NPC migration distance (2) the formation of the migration corona (3) cellular process orientation angle, and (4) neuronal migration. In our study we included the original compound EGCG, EGCG PEGylated-PLGA nanoparticles (Nano-EGCG), the polyphenolic compounds G37 and 
G56 with two galloyl units and a naphtyl or a biphenyl moiety, respectively, and M1 and M2 with one galloyl group (Figure 1). The inclusion of the latter was motivated by the results in our previous study where we observed that the natural catechin with only one galloyl group epigallocatechin (EGC, CAS: 970-74-1) disturbed the adhesion of NPCs less potently than EGCG (Barenys et al., 2017a). These five compounds: Nano-EGCG, G37, G56, M1, and M2, have already been tested for some beneficial effects related with, for example, their anticancer, anti-seizure, antibacterial and/or analgesic activity (Cano et al., 2018; Crous-Masó et al., 2018; Xifró et al., 2015), yet their potential to interfere with neurodevelopmental processes like migration needs evaluation before they are tested as prenatal therapies or prenatal nutritional supplements.

\section{Material and methods}

\subsection{Reagents}

(-)-Epigallocatechin-3-gallate (EGCG, CAS 989-51-5, purity > $98 \%$ ) was purchased from TransMIT PlantMetaChem (Giessen, Germany). 4-Hydroxy-2-naphtyl 3,4,5-trihydroxybenzoate (M1), 3hydroxy-1-naphtyl 3,4,5-trihydroxybenzoate $\quad$ (M2), $\quad$ 1,4-bis[(3,4,5trihidroxybenzol)oxy]naphthalene (G37) and 4,4'-bis[(3,4,5-trihidroxybenzol)oxy]-1,1'-biphenyl (G56) were synthesized as detailed by Crous-Masó et al., (2018) by the LIPPSO group at the University of Girona at a purity $>90 \%$. Stock solutions of EGCG and its four derivatives were dissolved at a final working concentration of $0.01 \%$ DMSO. 
Poly (lactic-co-glycolic acid)-polyethylene glycol (PLGA-PEG) 5\% was purchased from Evonik Co. (Evonik Co., Birmingham, USA). 1.5 mg/mL EGCG loaded PEGylated-PLGA nanoparticles were prepared at the laboratory of the 'Nanostructured systems for controlled drug delivery' research group as described in Cano et al., (2018). Briefly, the formulation with $1.5 \mathrm{mg} / \mathrm{ml}$ of EGCG, 14 $\mathrm{mg} / \mathrm{ml}$ of PLGA-PEG and Tween- ${ }^{\circledR} 801.5 \%$ led to a monodisperse population (polydispersity index: $\mathrm{PI}<0.1)$ of particles with an average particle size $\left(\mathrm{Z}_{\mathrm{av}}\right)$ of $168.5 \pm 9.9 \mathrm{~nm}$ and an encapsulation efficiency (EE) higher than 95\%. The formulation showed a zeta potential (ZP) value $(-23.3 \pm 5.3 \mathrm{mV})$. As the nanocarrier was formulated by the double emulsion method using Tween ${ }^{\circledR 80}$ as surfactant, working solutions of Nano-EGCG were prepared at a final concentration of $0.1 \%$ Tween ${ }^{\circledR} 80$.

\subsection{Ethics Statement}

The acquisition of brain tissue for preparation of neurospheres was approved by the Ethic Committee for Animal Experimentation of the University of Barcelona (CEEA) with license number OB 341/17.

\subsection{Proliferating cell culture establishment and maintenance}

See Figure 2 for a graphical representation of the experimental approach. Rat neural progenitor cells (NPCs) were isolated from whole brain of two postnatal day 1 (PND1) pups as previously described in Baumann et al., (2014). NPCs were cultivated as a floating culture in a humidified incubator at $37^{\circ} \mathrm{C}$ and $5 \% \mathrm{CO}_{2}$ in B27-proliferation medium [Dulbecco's modified Eagle medium (DMEM) and Hams F12 (3:1) with B27-supplement (Invitrogen GmBH, Karlsruhe, Germany), 20 $\mathrm{ng} / \mathrm{mL}$ Epidermal growth factor (EGF, Biosource, Karlsruhe, Germany), $10 \mathrm{ng} / \mathrm{mL}$ recombinant rat fibroblast growth factor (rrFGF, R\&D Systems, Wiesbaden-Nordenstadt, Germany), $100 \mathrm{U} / \mathrm{mL}$ penicillin and $100 \mu \mathrm{g} / \mathrm{mL}$ streptomycin (PAN-Biotech)]. Every 2-3 days half of the medium was replaced with fresh B27-proliferation medium. As soon as neurospheres reached a certain size of 0.5-0.6 $\mathrm{mm}$ in diameter they were passaged mechanically with a tissue chopper (Mclllwain) 
to maintain their proliferative character. NPCs were chopped 3-4 days before plating on a polyD-lysine/laminin-coated surface in an 8-chamber slide to perform the differentiation assay as described below.

\subsection{Differentiation conditions and chemical exposure}

Studies of migration and differentiation were performed under differentiation conditions as described elsewhere (Baumann et al., 2014). Briefly, NPCs were plated onto a coated surface of poly-D-lysine (PDL; $0.1 \mathrm{mg} / \mathrm{mL}$, Sigma Aldrich) and laminin $(5 \mu \mathrm{g} / \mathrm{mL}$, Sigma Aldrich) in an 8chamber slide filled with N2 differentiation medium [Dulbecco's modified Eagle medium (DMEM) and Hams F12 (3:1) supplemented with N2 (Invitrogen GmBH, Karlsruhe, Germany), $100 \mathrm{U} / \mathrm{mL}$ penicillin, and $100 \mu \mathrm{g} / \mathrm{mL}$ streptomycin (PAN-Biotech) in absence of the growth factors EGF and FGF]. 3-4 days after chopping five neurospheres of diameter $0.3 \mathrm{~mm}$ were placed on the surface of each chamber and exposed for $24 \mathrm{~h}$ to EGCG, Nano-EGCG, G37, G56, $\mathrm{M} 1$ or $\mathrm{M} 2$ at increasing concentrations $(1,5$ and $10 \mu \mathrm{M})$ at $37^{\circ} \mathrm{C}$ in a humidified $5 \% \mathrm{CO}_{2}$ incubator. Solvent controls were plated containing the same solvent concentrations then the tested compounds: $0.01 \%$ DMSO as solvent control for EGCG, G37, G56, M1 and M2 and $0.1 \%$ Tween ${ }^{\circledR} 80$ as solvent control for Nano-EGCG. EGF $(20 \mathrm{ng} / \mathrm{mL})$ was used as a positive control, since this concentration significantly increases migration and inhibits neuronal differentiation (Schmuck et al., 2017). Three to four independent experiments were performed for every endpoint and treatment.

\subsection{Migration}

All migration experiments were performed under differential conditions and were analyzed after an exposure time of $24 \mathrm{~h}$. To evaluate the individual endpoints the whole migration area of each neurosphere was visualized using a high performance phase-contrast microscope (Nikon Eclipse TS100) and pictures were taken with a monochrome camera (Imaging Source DMK 51AU02). 


\subsubsection{Migration distance}

The radial migration distance of each neurosphere was measured as described in Baumann et al., (2014) and Moors et al., (2007). Briefly, the radius in four perpendicular angles from the edge of the neurosphere core to the furthest migrated cells was measured by using the ImageJ software. Subsequently the mean of the four radii was calculated to obtain the migration distance of each sphere.

\subsubsection{Corona of migrating cells}

The corona of migrating cells around the sphere core was calculated by manual measurement of the angles of the area covered with cells of the total corona around a sphere core $\left(360^{\circ}\right)$. The sum of all angles around the neurospheres covered by cells was calculated.

\subsubsection{Orientation angle measurement}

NPCs migrate in a specific orientation angle within the migration area which was manually analyzed in two frames $(406.5 \mu \mathrm{m} \times 284.8 \mu \mathrm{m})$ per neurosphere. This orientation angle was measured in respect to the perpendicular direction from the sphere core for approximately 300 cell processes per concentration and experiment and subsequently plotted in a color-coded polar plot using MatLab R2017b version.

\subsection{Viability testing}

Viability was assessed directly after capturing pictures for the migration assay (24h after exposure) by means of Alamar Blue Assay using the CellTiter-Blue Reagent (CTB) (Promega, Mannheim, Germany) as described in Baumann et al., (2014).

\subsection{Immunocytochemistry}

After $24 \mathrm{~h}$ of exposure and subsequently viability testing cells were fixed overnight in $4 \%$ paraformaldehyde (PFA) at $4{ }^{\circ} \mathrm{C}$. On the following day cells were washed with $1 x$ Phosphate Buffered Saline (PBS) and stored in PBS at $4{ }^{\circ} \mathrm{C}$ until immunofluorescence staining was 
performed. To stain the cell nuclei and differentiated neurons within the migration area, neurospheres were incubated for $1 \mathrm{~h}$ at $37^{\circ} \mathrm{C}$ with $10 \%$ goat serum and rabbit-anti- $\beta$ (III)-tubulin first antibody (1:200; Sigma-Aldrich) in 1x Phosphate buffered saline with $0.1 \%$ (v/v) Triton-X 100 (PBS-T) followed by $30 \mathrm{~min}$ incubation at $37{ }^{\circ} \mathrm{C}$ with $2 \%$ goat serum, $1 \%$ Hoechst 33258 (Sigma-Aldrich) and anti-rabbit Alexa Fluor 546 secondary antibody (1:200; Sigma-Aldrich) in 1x PBS. Two images of the stained migrating area of each neurosphere were taken under a Nikon Eclipse TS100 fluorescence microscope. The number of Hoechst-positive cell nuclei was determined with the "Analyze Particle" command of ImageJ software (Rasband 1993-2007). BIIItubulin $^{+}$cells were quantified by manual counting in all images and expressed as percentage of the total number of cell nuclei within the two pictures corresponding to one neurosphere.

\subsection{Statistics}

Statistical analysis was performed with the Software GraphPad Prism, Version 7, by one-way ANOVA, followed by Bonferroni's test in the one compound concentration-response experiments. Two-way ANOVA, followed by Bonferroni's post hoc test was carried out to compare two compounds concentration-response curves. For each evaluated compound $50 \%$ effective concentrations $\left(\mathrm{EC}_{50}\right)$ were calculated by applying a nonlinear sigmoidal dose-response inhibition curve fit with variable slope to the obtained data points (with constrains top $=100$, bottom $=0$ ). Two-way ANOVA, followed by Bonferroni's post hoc test was also performed to determine the significance between angle-orientation variation and concentration. Student's ttest was used to compare the results between the two solvent controls and multiple student's t-test was applied to determine the significance between angle-orientation variation and concentration between the two solvent controls. Significance threshold in all statistical analysis was fixed at $p<0.05$. 


\section{Results}

In a previous study, EGCG exposure for $24 \mathrm{~h}$ disturbed migration of NPCs in both human and rat neurospheres (Barenys et al., 2017a). As there were no significant differences in sensitivity towards adverse EGCG effects between both species, we tested EGCG and five different relatedsubstances in only one model: the rat neurospheres. To follow the same approach of that study, we chose the same exposure period (24h), the same exposure concentration range (1 to $10 \mu \mathrm{M})$ and the same solvent control concentration $(0.01 \%$ DMSO) in all cases except in the nanoparticulated formulation Nano-EGCG where the solvent control needed to be $0.1 \%$ Tween ${ }^{\circledR 80}$, the surfactant used to ensure the optimal physicochemical characteristics of the developed nanosystem (Cano et al., 2018). As $0.1 \%$ Tween ${ }^{\circledR} 80$ was never used as solvent control in the 'Neurosphere Assay' before, we present in Supplementary Figure 1 the comparison of the raw values obtained for all evaluated endpoints for $0.01 \%$ DMSO and $0.1 \%$ Tween ${ }^{\circledR} 80$ controls, showing no statistically significant differences for any migration- or neuronal differentiationrelated endpoint and a significant increase in cell viability was detected by means of AlamarBlue assay in the $0.1 \%$ Tween ${ }^{\circledR} 80$ group which indicates that this solvent concentration is not cytotoxic for NPCs in culture.

The compounds selected to be included in this study were a nanoformulation of EGCG, and four EGCG structurally-related compounds including one (M1 and M2) or two (G37 and G56) galloyl groups (Figure 1). The tested compounds displayed different potencies in disturbing NPC migration distance after $24 \mathrm{~h}$ of exposure. G37 and M2 produced significant decreases of migration distance at $5 \mu \mathrm{M}, \mathrm{G} 56$ and $\mathrm{M} 1$ caused this effect at the maximum concentration tested $(10 \mu \mathrm{M})$, while Nano-EGCG did not significantly reduce the migration distance (Figure 3). G56, M1, M2 and Nano-EGCG displayed a significantly different concentration-response curve from the one of EGCG (Figure 3). 
Besides decreasing the migration distance, we observed the previously described characteristic effect of EGCG disturbing the migration pattern by induction of gaps and branches in the migration area (Figure 3). This effect, which so far has only been reported after exposure to catechin related compounds or to a functional beta1-integrin antibody, can be assessed by measuring the angle of the corona surrounding each neurosphere core with presence of migrating cells, irrespective of the distance of migration from the neurosphere core as published before for EGCG evaluation (Barenys et al., 2017a) and previously described in a different developmental neurotoxicity test using stem cells and measuring the neural outgrowth surrounding embryoid bodies (Theunissen et al., 2011). When the angle of the corona surrounded by migrating cells was measured, the tested compounds displayed very similar potencies than in the migration distance assay: G37 and M2 significantly decreased the angle of corona at $5 \mu \mathrm{M}$, while G56 and M1 only presented significant effects at $10 \mu \mathrm{M}$ and Nano-EGCG did not induce a significant reduction of the endpoint (Figure 3). Also in this case, when concentration-response curves of each compound were compared to the one of EGCG, all tested compounds displayed a significantly different curve profile than EGCG except G37 (Figure 3).

To better compare the potencies of the effects among all compounds, $50 \%$ effective concentrations $\left(\mathrm{EC}_{50}\right)$ were calculated for the two previously presented endpoints as well as for viability results by applying a nonlinear sigmoidal dose response curve fit to the obtained data points (Figure 4). $\mathrm{EC}_{50}$ values were above the tested concentrations for all endpoints for $\mathrm{G} 56$ and Nano-EGCG. For M1, EC 50 values were also above the tested concentrations for all endpoints except for migration where it was $8.61 \mu \mathrm{M}$. In contrast, $\mathrm{G} 37$ presented $\mathrm{EC}_{50}$ values of the same magnitude than EGCG while M2 displayed approximately two times higher $\mathrm{EC}_{50}$ values for the endpoints migration distance and corona formation. Viability curves representing metabolic activity measured with the Alamar Blue Assay showed that $\mathrm{EC}_{50}$ values were not reached in any compound except for EGCG and G37. A very recent study shows that migration distance or pattern can define the magnitude of signal of viability assays like the Alamar Blue Assay because 
the metabolizing rate is related to number of cells in the migration area (Fritsche et al., 2018). Therefore it is not surprising that EGCG and G37, the two compounds with the strongest effect in migration distance and migration pattern are the only ones reaching an $\mathrm{EC}_{50}$ in the Alamar Blue Assay. In that study, to discard a direct cytotoxic effect of EGCG in NPCs a different assay not directly dependent on cell number was applied: FACS analyses identifying annexinV/PI positive cells clearly showed that EGCG does not cause cell death (Fritsche et al., 2018).

A complementary approach to evaluate the effect of EGCG on the migration of NPCs is to measure the orientation angle of the cell migrating processes in a fixed area. As cells migrate radially from the neurosphere core, cellular processes of solvent control neurospheres were mainly oriented between $0^{\circ}$ and $\pm 30^{\circ}$ angles toward the reference axis (for a schematic representation of the measurement method the reader is referred to Barenys et al., 2017a). In contrast, as previously observed, $24 \mathrm{~h}$ EGCG exposure caused a chaotic orientation of processes with a significantly increased percentage of cells between $\pm 30^{\circ}$ and $\pm 60^{\circ}$ angles. In this case, at higher concentrations, a significant increase in the percentage of cells oriented between $\pm 60^{\circ}$ and $\pm 90^{\circ}$ angles was also observed, while Nano-EGCG did not change the orientation of cell processes at any tested concentration (Figure 5).

For the other four tested compounds, in all cases the disturbance effect induced was less potent than after exposure to EGCG, as no significant effects were observed at $1 \mu \mathrm{M}$ and there was no significant increase in the percentage of cells oriented between $\pm 60^{\circ}$ and $\pm 90^{\circ}$ angles after exposure to any of the compounds tested. Again, when comparing among them four, the effect was more potent after exposure to $\mathrm{G} 37$ and $\mathrm{M} 2$ than after exposure to $\mathrm{G} 56$ or $\mathrm{M} 1$, which was not inducing significant effects until $10 \mu \mathrm{M}$.

As in the 'Neurosphere Assay' the radial glial scaffold is normally used by young neurons to migrate away from the sphere core, we finally evaluated if the alterations in cell orientation had 
an impact in the percentage of migrating neurons, as previously observed with EGCG in human NPC (Barenys et al., 2017a). Similar to previous results in human neurospheres, after $24 \mathrm{~h}$ of exposure, EGCG induced a significant reduction in the percentage of neurons in the migration area also of rat neurospheres only at the highest concentration tested ( $10 \mu \mathrm{M}$; Figure 6) (Barenys et al., 2017a). From all other compounds tested, only $M 2$ induced a significant decrease in the percentage of neurons at concentrations even lower than EGCG $(1 \mu \mathrm{M})$, while G37 and M1 showed a trend to reduction without reaching statistical significance (ANOVA $p$ value $=0.06$ and 0.07 respectively). Again, Nano-EGCG and G56 had no adverse effect on the endpoint studied at any concentration.

\section{Discussion}

The within-laboratory variability of the results obtained with the 'Neurosphere Assay' in control or exposed neurospheres has already been published in several articles performed by different operators over a long time (Baumann et al., 2016; Fritsche et al., 2005; Moors et al., 2009; Schmuck et al., 2017). In addition historical control values of the different endpoints of the neurosphere assay were summarized (Baumann et al., 2015). But so far, the transferability of the method in a second laboratory was not shown. In the present work, we reproduce the results previously published for solvent control (DMSO 0.01\%) and EGCG exposed neurospheres in a different laboratory (University of Barcelona) of a different country (Spain), with different equipment and using neurospheres gained from a different rat strain (Sprague-Dawley). The values obtained in control culture conditions in this new lab fulfill the quality requirements previously published (Baumann et al., 2016, 2014) and EGCG exposure disturbed NPC migration by inducing the same pattern of alterations: significantly reduced migration distance, significantly decreased angle of corona with presence of migrating cells, significantly disturbed orientation of migrating cells' processes, and at higher concentrations reduced neuronal migration. The transferability of results among laboratories is a requirement of the retrospective 
validation process of new methods in toxicology described by Hartung et al., (2004), which outlines the path to evaluate the validity of a new test on the basis of a modular validation approach and which was already used for example to retrospectively validate the in vitro micronucleus test (Corvi et al., 2008). According to the recommendations on test readiness criteria for new approach methods in toxicology (Bal-Price et al., 2018) the present results contribute to prove the robustness of the 'Neurosphere Assay' (criteria 9c-interlab) and bring forward its readiness.

The main aim of this study was to compare the effects of EGCG on NPC migration with the ones induced by the structurally related compounds G37, G56, M1, M2 and Nano-EGCG, developed as new therapeutical strategies. The overall evaluation of the endpoints shows a consistent pattern of different potencies of the tested compounds:

$$
\text { EGCG } \approx \text { G37 }>\text { M2 }>\text { M1 } \approx \text { G56 }>\text { Nano-EGCG }
$$

Several studies have shown that EGCG can bind to laminin (Peter et al., 2017; Suzuki and Isemura, 2001) and disturb the adhesion of cells to the extracellular matrix (Bracke et al., 1987; Chen et al., 2003; Suzuki and Isemura, 2001) finally leading to an altered cell migration pattern (Bal-Price et al., 2017; Barenys et al., 2017a; Nyffeler et al., 2017). The structural moieties inducing this altered cell migration seemed to be galloyl/pyrogallol groups because in a previous study, catechins lacking these residues did not disrupt cell adhesion (Barenys et al., 2017a). In that study we postulated that compounds with only one galloyl or pyrogallol moiety would disturb NPC migration less potently than compounds including two of them. With the results of the present work we can refute this hypothesis, as M2 with only one galloyl moiety disturbs migration more potently $\left(\mathrm{EC}_{50 \mathrm{migration}-\mathrm{M} 2}=3.06 \mu \mathrm{M}\right)$ than $\mathrm{G} 56$ with two $\left(\mathrm{EC}_{50 \mathrm{migration}-\mathrm{G} 56}=\right.$ not reached). The current results also indicate that variations in the main structure can cause large differences in migration disturbance potency as G37 and G56 include both two galloyl groups 
but G37 with a naphthyl unit disturbs migration more potently than G56 with a biphenyl one $\left(\mathrm{EC}_{50 \text { migration-G37 }}=2.17 \mu \mathrm{M} \ll<\mathrm{EC}_{50 \text { migration-G56 }}=\right.$ not reached $)$.

Hence, the structural interplay between galloyl groups and other aromatic ring groups within the same molecule seems to determine laminin binding capacity that masks integrin binding sites. To unravel the precise structures necessary for exerting these effects, more compounds structurally related to EGCG need to be tested in this assay.

To compare the EGCG-related compounds' potencies at an in vivo relevant concentration-range all compounds were tested between 1 and $10 \mu \mathrm{M}$ (reviewed by Barenys et al., 2017b). Concentrations of EGCG in human plasma within this range have already been described after EGCG supplements intake (Shanafelt et al., 2009; Ullmann et al., 2003), however, a 5 or $10 \mu \mathrm{M}$ concentration would not be achievable in individuals with a non-supplemented diet (Skibola and Smith, 2000). When tested at this range of concentrations, compounds $G 37$ and M2 disturbed some endpoints with an $\mathrm{EC}_{50}<5 \mu \mathrm{M}$. G56 and $\mathrm{M} 1$ significantly disturbed migration distance and the formation of the migration corona only at $10 \mu \mathrm{M}$ and for $\mathrm{G}_{6} 6 \mathrm{EC}_{50}$ values were not reached within the tested concentrations. For these compounds, concentrations around $10 \mu \mathrm{M}$ are not achieved in the developing brain in vivo, as pharmacokinetic studies in pregnant rats indicate that for example total EGCG is found at approximately eight times lower concentrations in the fetal brain than in maternal plasma (Chu et al., 2006). However, pharmacokinetic studies in pregnant animals with G37, G56, M1, M2 or Nano-EGCG have not been performed yet. In this sense, even if $10 \mu \mathrm{M}$ EGCG already induce a total response in migration, and higher concentrations would exceed in vivo relevance, the addition of a $20 \mu \mathrm{M}$ concentration for some of the other tested compounds would have strengthened the calculation of $\mathrm{EC}_{50}$ values. According to our results, M1 and G56 would seem good candidates to develop therapeutic or preventive strategies for pregnant women, however, M1 induces a non-significant decrease in migration of young neurons after $24 \mathrm{~h}(\mathrm{p}=0.07)$ which could be expected to become stronger 
after longer exposures. As G56 does not induce any reduction in this endpoint either, G56 would seem a better alternative than M1. Other studies have shown that G56 is significantly more effective than EGCG in reducing thermal hyperalgesia in a chronic constriction nerve injury mice model, that it displays strongest inhibition of lipogenic enzyme fatty acid synthase (FASN) than EGCG and that compared to the control group it does not cause significant changes on body weight neither alterations in hepatic, renal or hematological function markers at daily doses of 50 and $75 \mathrm{mg} / \mathrm{Kg}$ (Xifró et al., 2015).

Furthermore, the nanoformulation of EGCG did not disturb any of the evaluated endpoints in this study ( $\mathrm{EC}_{50 \mathrm{~s}}=$ not reached for any endpoint). It is expected that in this particular case EGCG presents a more sustained release to the medium than when neurospheres are exposed to the free drug as this is precisely one of the aims of nanoencapsulating a compound, to contribute to a slower delivery of the drug. This sustained delivery might be responsible of a decreased concentration in the medium and a decreased interaction of the compound with the cells of the sphere or with the extracellular matrix, and therefore be responsible of a decrease or in this case an avoidance of adverse effects. The fact that a different solvent control was used while testing Nano-EGCG $\left(0.1 \%\right.$ Tween $\left.{ }^{\circledR} 80\right)$ did not influence the outcome, as the results of this solvent control are comparable with the ones of the solvent control used for G37, G56, M1 and M2 (0.01\% DMSO; Supplementary Figure 1). This comparison also contributes to identify Tween ${ }^{\circledR} 80$ as a usable solvent control in the 'Neurosphere Assay', which might be of relevance due to its common use to develop nanoparticulated systems. Based on our results, Nano-EGCG could also be a potentially useful alternative to EGCG for developmental therapies. Previously published studies showed that Nano-EGCG significantly reduced the number and the intensity of epileptic episodes in mice more than the administration of the free drug (Cano et al., 2018).

Finally, it is important to remark that compounds containing galloyl moieties should be suspected to disturb the neurodevelopmental key event 'NPC migration', and therefore, 
developmental neurotoxicity relevant evaluations should be included in the process of developing new medical drugs structurally-related with this family.

In summary, we can conclude that several compounds structurally-related to EGCG that are designed as its new therapeutical alternatives, induce the same pattern of alterations in the migration of NPCs than EGCG and some of them do it with the same potency (G37). In general, the potency of the effects of these compounds does not necessarily depend on the number of galloyl groups present in the molecule. This study suggests G56 and Nano-EGCG as potential therapeutic or preventive alternatives to EGCG for the prenatal period but further comprehensive evaluations of the neurodevelopmental effects of these compounds are needed to confirm their safety.

\section{Acknowledgements}

The authors acknowledge the financial support from the University of Girona (MPCUdG2016/036) and the support of the Catalonian government (2017SGR00385). This work was also supported partially by Spanish grants from Fundación Ramón Areces and Instituto de Salud Carlos III (PI1400329). B.K. acknowledges the support of the Erasmus+ EU Programme and A.C. acknowledges the support of the Generalitat de Catalunya for the PhD scholarship FI-DGR (CVE-DOGC-B-14206020-2014).

\section{Conflicts of Interest}

The authors declare no conflict of interest.

\section{References}

Bal-Price, A., Hogberg, H.T., Crofton, K.M., Daneshian, M., FitzGerald, R.E., Fritsche, E., 
Heinonen, T., Hougaard Bennekou, S., Klima, S., Piersma, A.H., Sachana, M., Shafer, T.J., Terron, A., Monnet-Tschudi, F., Viviani, B., Waldmann, T., Westerink, R.H.S., Wilks, M.F., Witters, H., Zurich, M.G., Leist, M., 2018. Recommendation on test readiness criteria for new approach methods in toxicology: Exemplified for developmental neurotoxicity. ALTEX. https://doi.org/10.14573/altex.1712081

Bal-Price, A., Lein, P.J., Keil, K.P., Sethi, S., Shafer, T., Barenys, M., Fritsche, E., Sachana, M., Meek, M.E., 2017. Developing and applying the adverse outcome pathway concept for understanding and predicting neurotoxicity. Neurotoxicology 59, 240-255. https://doi.org/10.1016/j.neuro.2016.05.010

Barenys, M., Gassmann, K., Baksmeier, C., Heinz, S., Reverte, I., Schmuck, M., Temme, T., Bendt, F., Zschauer, T.-C., Rockel, T.D., Unfried, K., Wätjen, W., Sundaram, S.M., Heuer, H., Colomina, M.T., Fritsche, E., 2017a. Epigallocatechin gallate (EGCG) inhibits adhesion and migration of neural progenitor cells in vitro. Arch. Toxicol. 91. https://doi.org/10.1007/s00204-016-1709-8

Barenys, M., Masjosthusmann, S., Fritsche, E., 2017b. Is intake of flavonoid-based food supplements during pregnancy safe for the developing child? A literature review. Curr. Drug Targets 18. https://doi.org/10.2174/1389450116666150804110049

Baumann, J., Barenys, M., Gassmann, K., Fritsche, E., 2014. Comparative human and rat "neurosphere assay" for developmental neurotoxicity testing. Curr. Protoc. Toxicol. 1. https://doi.org/10.1002/0471140856.tx1221s59

Baumann, J., Dach, K., Barenys, M., Giersiefer, S., Goniwiecha, J., Lein, P.J., Fritsche, E., 2015. Application of the Neurosphere Assay for DNT Hazard Assessment: Challenges and Limitations. Humana Press, Totowa, NJ, pp. 1-29. https://doi.org/10.1007/7653_2015_49 LB - ref1 
Baumann, J., Gassmann, K., Masjosthusmann, S., DeBoer, D., Bendt, F., Giersiefer, S., Fritsche, E., 2016. Comparative human and rat neurospheres reveal species differences in chemical effects on neurodevelopmental key events. Arch Toxicol 90, 1415-1427. https://doi.org/10.1007/s00204-015-1568-8

Bracke, M.E., Castronovo, V., Van Cauwenberge, R.M., Coopman, P., Vakaet, L.J., Strojny, P., Foidart, J.M., Mareel, M.M., 1987. The anti-invasive flavonoid (+)-catechin binds to laminin and abrogates the effect of laminin on cell morphology and adhesion. Exp. Cell Res. 173, 193-205.

Cano, A., Ettcheto, M., Espina, M., Auladell, C., Calpena, A.C., Folch, J., Barenys, M., SánchezLópez, E., Camins, A., García, M.L., 2018. Epigallocatechin-3-gallate loaded PEGylatedPLGA nanoparticles: A new anti-seizure strategy for temporal lobe epilepsy. Nanomedicine Nanotechnology, Biol. Med. 14. https://doi.org/10.1016/j.nano.2018.01.019

Chen, C.-N., Liang, C.-M., Lai, J.-R., Tsai, Y.-J., Tsay, J.-S., Lin, J.-K., 2003. Capillary electrophoretic determination of theanine, caffeine, and catechins in fresh tea leaves and oolong tea and their effects on rat neurosphere adhesion and migration. J. Agric. Food Chem. 51, 7495-7503. https://doi.org/10.1021/jf034634b

Chu, K.O., Wang, C.C., Chu, C.Y., Chan, K.P., Rogers, M.S., Choy, K.W., Pang, C.P., 2006. Pharmacokinetic studies of green tea catechins in maternal plasma and fetuses in rats. J. Pharm. Sci. 95, 1372-1381. https://doi.org/10.1002/jps.20594

Corvi, R., Albertini, S., Hartung, T., Hoffmann, S., Maurici, D., Pfuhler, S., van Benthem, J., Vanparys, P., 2008. ECVAM retrospective validation of in vitro micronucleus test (MNT). Mutagenesis 23, 271-283. https://doi.org/10.1093/mutage/gen010

Crous-Masó, J., Palomeras, S., Relat, J., Camó, C., Martínez-Garza, Ú., Planas, M., Feliu, L., Puig, 
T., 2018. (-)-Epigallocatechin 3-gallate synthetic analogues inhibit fatty acid synthase and show anticancer activity in triple negative breast cancer. Molecules 23.

https://doi.org/10.3390/molecules23051160

Fritsche, E., Barenys, M., Klose, J., Masjosthusmann, S., Nimtz, L., Schmuck, M., Wuttke, S., Tigges, J., 2018. Current availability of stem cell-based in vitro methods for Developmental Neurotoxicity (DNT) testing. Toxicol. Sci. kfy178. https://doi.org/10.1093/toxsci/kfy178

Fritsche, E., Cline, J.E., Nguyen, N.-H., Scanlan, T.S., Abel, J., 2005. Polychlorinated biphenyls disturb differentiation of normal human neural progenitor cells: clue for involvement of thyroid hormone receptors. Environ. Health Perspect. 113, 871-876.

Hartung, T., Bremer, S., Casati, S., Coecke, S., Corvi, R., Fortaner, S., Gribaldo, L., Halder, M., Hoffmann, S., Roi, A.J., Prieto, P., Sabbioni, E., Scott, L., Worth, A., Zuang, V., 2004. A modular approach to the ECVAM principles on test validity. Altern. Lab. Anim. 32, 467472.

Hollman, P.C., Feskens, E.J., Katan, M.B., 1999. Tea flavonols in cardiovascular disease and cancer epidemiology. Proc. Soc. Exp. Biol. Med. 220, 198-202.

Holmes, G.L., McCabe, B., 2001. Brain development and generation of brain pathologies. Int. Rev. Neurobiol. 45, 17-41.

Kennedy, D.A., Lupattelli, A., Koren, G., Nordeng, H., 2013. Herbal medicine use in pregnancy : results of a multinational study.

Krupkova, O., Ferguson, S.J., Wuertz-Kozak, K., 2016. Stability of (-)-epigallocatechin gallate and its activity in liquid formulations and delivery systems. J. Nutr. Biochem. 37, 1-12. https://doi.org/10.1016/j.jnutbio.2016.01.002

Long, L., Li, Y., Wang, Y.D., He, Q.Y., Li, M., Cai, X.D., Peng, K., Li, X.P., Xie, D., Wen, Y.L., Yin, 
D.L., Peng, Y., 2010. The preventive effect of oral EGCG in a fetal alcohol spectrum disorder mouse model. Alcohol. Clin. Exp. Res. 34, 1929-1936.

https://doi.org/10.1111/j.1530-0277.2010.01282.x

McElyea, S.D., Starbuck, J.M., Tumbleson-Brink, D.M., Harrington, E., Blazek, J.D., Ghoneima, A., Kula, K., Roper, R.J., 2016. Influence of prenatal EGCG treatment and Dyrk1a dosage reduction on craniofacial features associated with Down syndrome. Hum. Mol. Genet. 25, 4856-4869. https://doi.org/10.1093/hmg/ddw309

Mineva, N.D., Paulson, K.E., Naber, S.P., Yee, A.S., Sonenshein, G.E., 2013. Epigallocatechin-3gallate inhibits stem-like inflammatory breast cancer cells. PLoS One 8, e73464. https://doi.org/10.1371/journal.pone.0073464

Moors, M., Cline, J.E., Abel, J., Fritsche, E., 2007. ERK-dependent and -independent pathways trigger human neural progenitor cell migration. Toxicol Appl Pharmacol 221, 57-67. https://doi.org/10.1016/j.taap.2007.02.018

Moors, M., Rockel, T.D., Abel, J., Cline, J.E., Gassmann, K., Schreiber, T., Schuwald, J., Weinmann, N., Fritsche, E., 2009. Human neurospheres as three-dimensional cellular systems for developmental neurotoxicity testing. Env. Heal. Perspect 117, 1131-1138. https://doi.org/10.1289/ehp.0800207

Nyffeler, J., Dolde, X., Krebs, A., Pinto-Gil, K., Pastor, M., Behl, M., Waldmann, T., Leist, M., 2017. Combination of multiple neural crest migration assays to identify environmental toxicants from a proof-of-concept chemical library. Arch. Toxicol. 91, 3613-3632. https://doi.org/10.1007/s00204-017-1977-y

Ortiz-Romero, P., Borralleras, C., Bosch-Morato, M., Guivernau, B., Albericio, G., Munoz, F.J., Perez-Jurado, L.A., Campuzano, V., 2018. Epigallocatechin-3-gallate improves cardiac hypertrophy and short-term memory deficits in a Williams-Beuren syndrome mouse 
model. PLoS One 13, e0194476. https://doi.org/10.1371/journal.pone.0194476

Peter, B., Farkas, E., Forgacs, E., Saftics, A., Kovacs, B., Kurunczi, S., Szekacs, I., Csampai, A., Bosze, S., Horvath, R., 2017. Green tea polyphenol tailors cell adhesivity of RGD displaying surfaces: multicomponent models monitored optically. Sci. Rep. 7, 42220. https://doi.org/10.1038/srep42220

Puig, T., Turrado, C., Benhamú, B., Aguilar, H., Relat, J., Ortega-Gutiérrez, S., Casals, G., Marrero, P.F., Urruticoechea, A., Haro, D., López-Rodríguez, M.L., Colomer, R., 2009. Novel inhibitors of fatty acid synthase with anticancer activity. Clin. Cancer Res. 15, 76087615. https://doi.org/10.1158/1078-0432.CCR-09-0856

Rothwell, J.A., Perez-Jimenez, J., Neveu, V., Medina-Remon, A., M'hiri, N., Garcia-Lobato, P., Manach, C., Knox, C., Eisner, R., Wishart, D.S., Scalbert, A., 2013. Phenol-Explorer 3.0: a major update of the Phenol-Explorer database to incorporate data on the effects of food processing on polyphenol content. Database (Oxford). 2013, bat070. https://doi.org/10.1093/database/bat070

Schmuck, M.R., Temme, T., Dach, K., de Boer, D., Barenys, M., Bendt, F., Mosig, A., Fritsche, E., 2017. Omnisphero: a high-content image analysis (HCA) approach for phenotypic developmental neurotoxicity (DNT) screenings of organoid neurosphere cultures in vitro. Arch Toxicol 91, 2017-2028. https://doi.org/10.1007/s00204-016-1852-2

Shanafelt, T.D., Call, T.G., Zent, C.S., LaPlant, B., Bowen, D.A., Roos, M., Secreto, C.R., Ghosh, A.K., Kabat, B.F., Lee, M.-J., Yang, C.S., Jelinek, D.F., Erlichman, C., Kay, N.E., 2009. Phase I trial of daily oral Polyphenon E in patients with asymptomatic Rai stage 0 to II chronic lymphocytic leukemia. J. Clin. Oncol. 27, 3808-3814.

https://doi.org/10.1200/JCO.2008.21.1284

Shankar, S., Ganapathy, S., Hingorani, S.R., Srivastava, R.K., 2008. EGCG inhibits growth, 
invasion, angiogenesis and metastasis of pancreatic cancer. Front. Biosci. 13, 440-452.

Singh, B.N., Shankar, S., Srivastava, R.K., 2011. Green tea catechin, epigallocatechin-3-gallate (EGCG): mechanisms, perspectives and clinical applications. Biochem. Pharmacol. 82, 1807-1821. https://doi.org/10.1016/j.bcp.2011.07.093

Skibola, C.F., Smith, M.T., 2000. Potential health impacts of excessive flavonoid intake. Free Radic. Biol. Med. 29, 375-383.

Suzuki, Y., Isemura, M., 2001. Inhibitory effect of epigallocatechin gallate on adhesion of murine melanoma cells to laminin. Cancer Lett. 173, 15-20.

Theunissen, P.T., Pennings, J.L.A., Robinson, J.F., Claessen, S.M.H., Kleinjans, J.C.S., Piersma, A.H., 2011. Time-Response Evaluation by Transcriptomics of Methylmercury Effects on Neural Differentiation of Murine Embryonic Stem Cells. Toxicol. Sci. 122, 437-447. https://doi.org/10.1093/toxsci/kfr134

Tiwari, V., Kuhad, A., Chopra, K., 2010. Epigallocatechin-3-gallate ameliorates alcohol-induced cognitive dysfunctions and apoptotic neurodegeneration in the developing rat brain. Int. J. Neuropsychopharmacol. 13, 1053-1066. https://doi.org/10.1017/S146114571000060X

Turrado, C., Puig, T., García-Cárceles, J., Artola, M., Benhamú, B., Ortega-Gutiérrez, S., Relat, J., Oliveras, G., Blancafort, A., Haro, D., Marrero, P.F., Colomer, R., López-Rodríguez, M.L., 2012. New synthetic inhibitors of fatty acid synthase with anticancer activity. J. Med. Chem. 55, 5013-5023. https://doi.org/10.1021/jm2016045

Ullmann, U., Haller, J., Decourt, J.P., Girault, N., Girault, J., Richard-Caudron, A.S., Pineau, B., Weber, P., 2003. A single ascending dose study of epigallocatechin gallate in healthy volunteers. J. Int. Med. Res. 31, 88-101. https://doi.org/10.1177/147323000303100205

WS, R., 1993-2007, n.d. ImageJ. https://doi.org/http://imagej.nih.gov/ij/ 
Xifró, X., Vidal-Sancho, L., Boadas-Vaello, P., Turrado, C., Alberch, J., Puig, T., Verdú, E., 2015. Novel epigallocatechin-3-gallate (EGCG) derivative as a new therapeutic strategy for reducing neuropathic pain after chronic constriction nerve injury in mice. PLoS One 10,115. https://doi.org/10.1371/journal.pone.0123122

Yang, C.S., Wang, X., Lu, G., Picinich, S.C., 2009. Cancer prevention by tea: animal studies, molecular mechanisms and human relevance. Nat. Rev. Cancer 9, 429-439.

https://doi.org/10.1038/nrc2641 
Figures and Figure legends:<smiles>O=C(OC1Cc2c(O)cc(O)cc2OC1c1cc(O)c(O)c(O)c1)c1cccc(O)c1</smiles>

EGCG

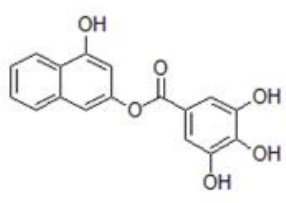

M1

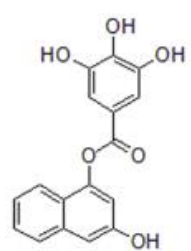

M2

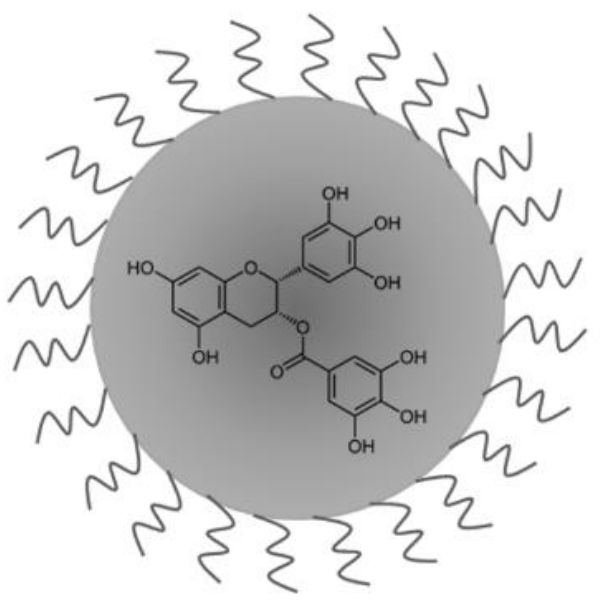

EGCG PEGylated-

PLGA Nanoparticles

(Nano-EGCG)

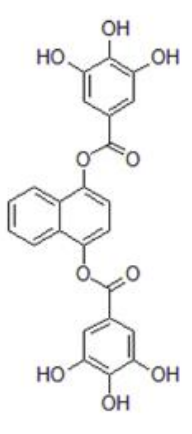

G37

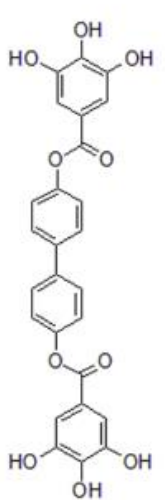

G56

Figure 1. Chemical structure of the tested compounds. EGCG (458.375 g/mol): epigallocatechin gallate; M1 (312.27 g/mol): 4-hydroxy-2-naphthyl 3,4,5-trihydroxybenzoate; M2 (312.27 g/mol): 3-hydroxy-1naphthyl 3,4,5-trihydroxybenzoate; EGCG PEGylated-PLGA Nanoparticles (Nano-EGCG); G37 (464.38 g/mol): 1,4-bis[(3,4,5-trihydroxybenzoyl)oxy]naphthalene; G56 (490.42 g/mol): 4,4'-bis[(3,4,5trihydroxybenzoyl)oxy]-1,1'-biphenyl. 


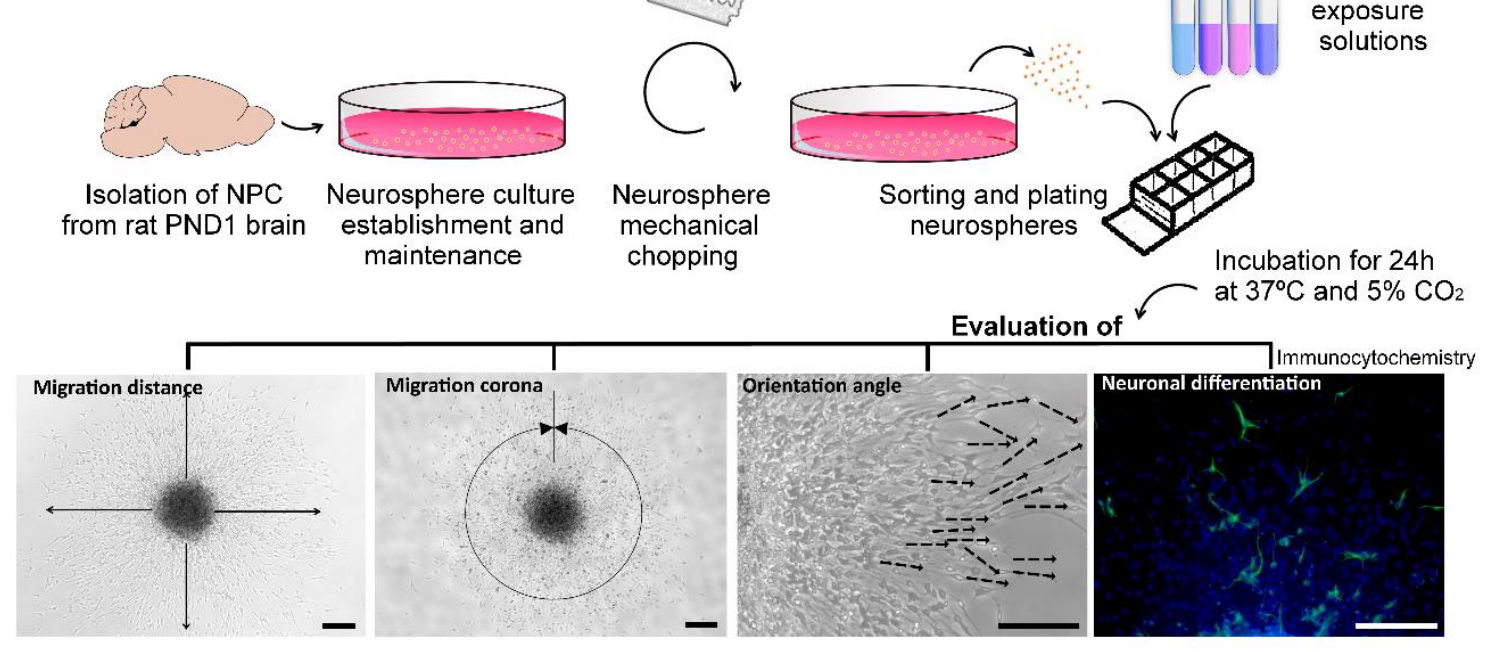

Figure 2. Graphical representation of the experimental approach. Migration distance. Phase-contrast image of the whole migration area of a rat neurosphere after $24 \mathrm{~h}$ of culture on a PDL/laminin coated surface. Migration distance measurement in 4 perpendicular radii from the neurosphere edge to the furthest migrated cells is exemplified with 4 arrows. Migration corona. Phase-contrast image of the whole migration area of a rat neurosphere after $24 \mathrm{~h}$ of culture on a PDL/laminin coated surface. The angle of the corona with presence of migrating cells surrounding the neurosphere core is represented with an arrow. Orientation angle. Phase-contrast image of the right side of the migration area of a rat neurosphere after $24 \mathrm{~h}$ of culture on a PDL/laminin coated surface. Measurement of 20 orientation angles is represented with discontinuous arrows. Neuronal differentiation. Fluorescent microscope image of the upper part of the migration area of a rat neurosphere after $24 \mathrm{~h}$ of culture on a PDL/laminin coated surface, fixation and immunocytochemistry. Blue: nuclei staining (Hoechst), green: neuronal marker ( $\beta$ IIItubulin). Scale bars: $100 \mu \mathrm{m}$. 
A

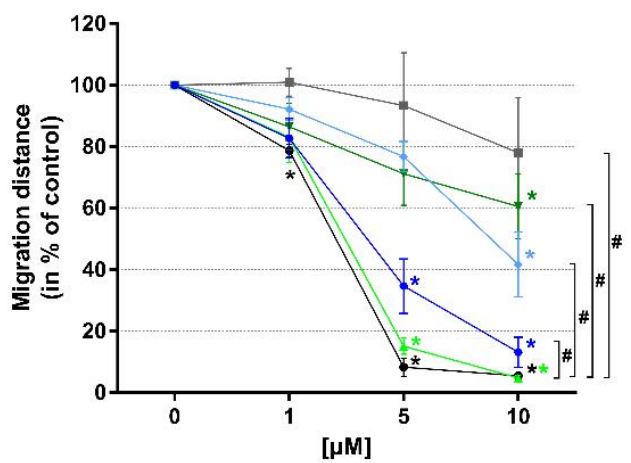

B
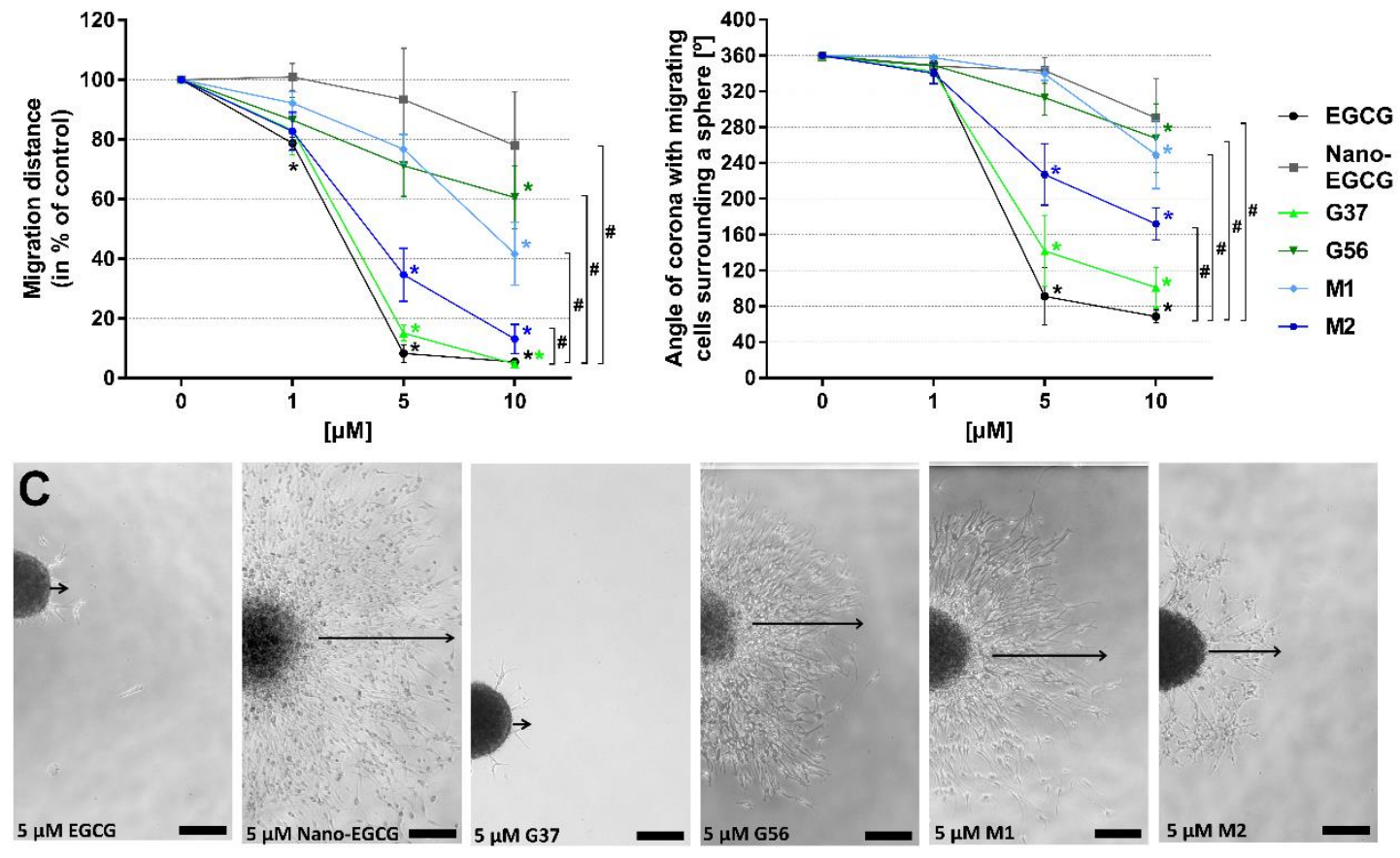

D
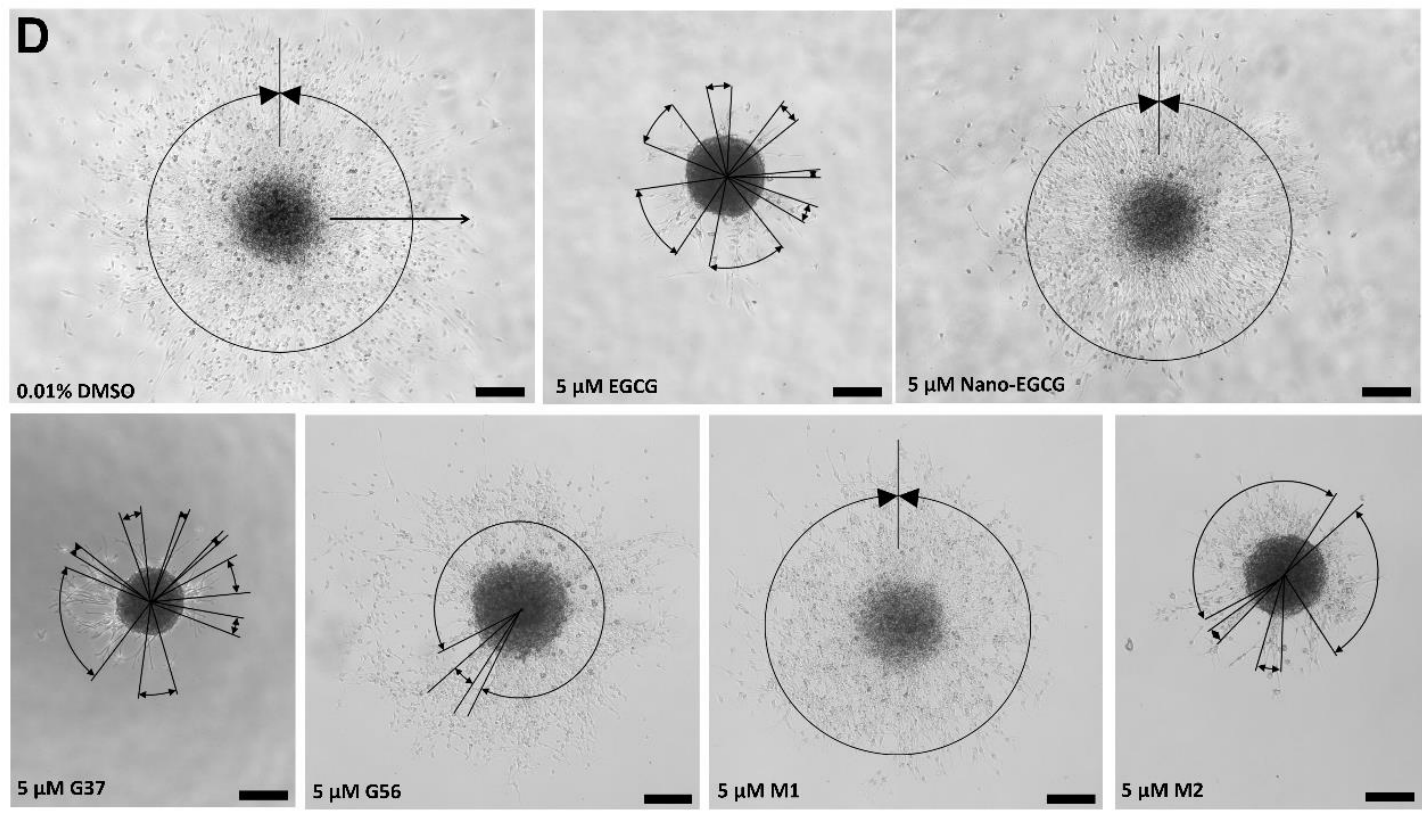

Figure 3. EGCG, Nano-EGCG and structurally related compounds display different potencies in disturbing NPC migration distances and the formation of the migration corona. Rat neurospheres were cultured for $24 \mathrm{~h}$ with increasing concentrations of EGCG, Nano-EGCG, G37, G56, M1 and M2. Migration distance $(\mathbf{A})$ and the angle of the corona with presence of migrating cells surrounding the neurosphere core (B) were evaluated in five neurospheres/concentration in four independent experiments. Results are presented as mean \pm SEM in $\%$ of respective solvent control (DMSO $0.01 \%$ for EGCG, G37, G56, M1 and M2 or $0.1 \%$ Tween ${ }^{\circledR} 80$ for Nano-EGCG), and mean \pm SEM angle values in degrees, respectively. ${ }^{*} p<0.05$ 
versus respective solvent control by one-way ANOVA and Bonferroni test. \#p<0.05 versus EGCG concentration-response curve by two-way ANOVA. C. Representative phase-contrast images of neurospheres exposed to $5 \mu \mathrm{M}$ of each test compound for $24 \mathrm{~h}$ showing part of their migration area with one of the measured distances indicated with an arrow. D. Representative phase-contrast images of neurospheres exposed to solvent control $0.01 \%$ DMSO, or $5 \mu \mathrm{M}$ of all tested compounds for $24 \mathrm{~h}$ showing the full migration area and the measured angles of corona with migrating cells around a sphere (In solvent control picture $D$, an arrow indicating the measured migration distance is also included for comparison with C). Scale bars: $100 \mu \mathrm{m}$. 

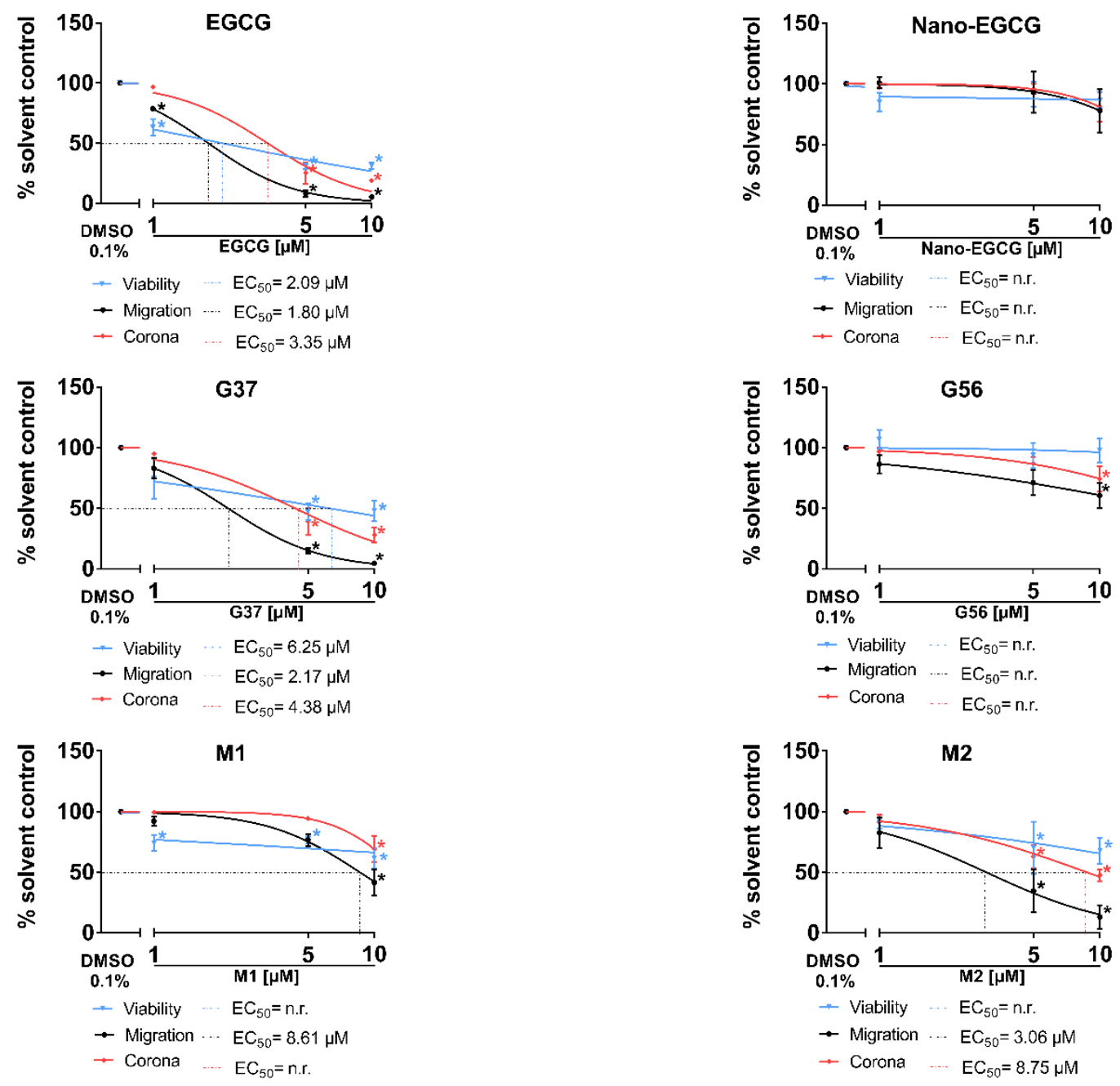

Figure 4. EGCG structurally related compounds display different potencies in disturbing NPCs migration related endpoints. Rat neurospheres were cultured for $24 \mathrm{~h}$ with increasing concentrations of EGCG, Nano-EGCG, G37, G56, M1 and M2. Blue (viability), black (migration) and orange lines (angle of corona with migrating cells) represent the nonlinear sigmoidal dose response curve fitted to the data points of four independent experiments using GraphPad Prism v7. Data points represent the mean \pm SEM in $\%$ of respective solvent control (DMSO $0.01 \%$ for EGCG, G37, G56, M1 and M2 or $0.1 \%$ Tween ${ }^{\circledR} 80$ for NanoEGCG). n.r.: not reached. ${ }^{*} p<0.05$ versus respective solvent control by one-way ANOVA and Bonferroni test. 
Solvent Control $1 \boldsymbol{\mu M}$

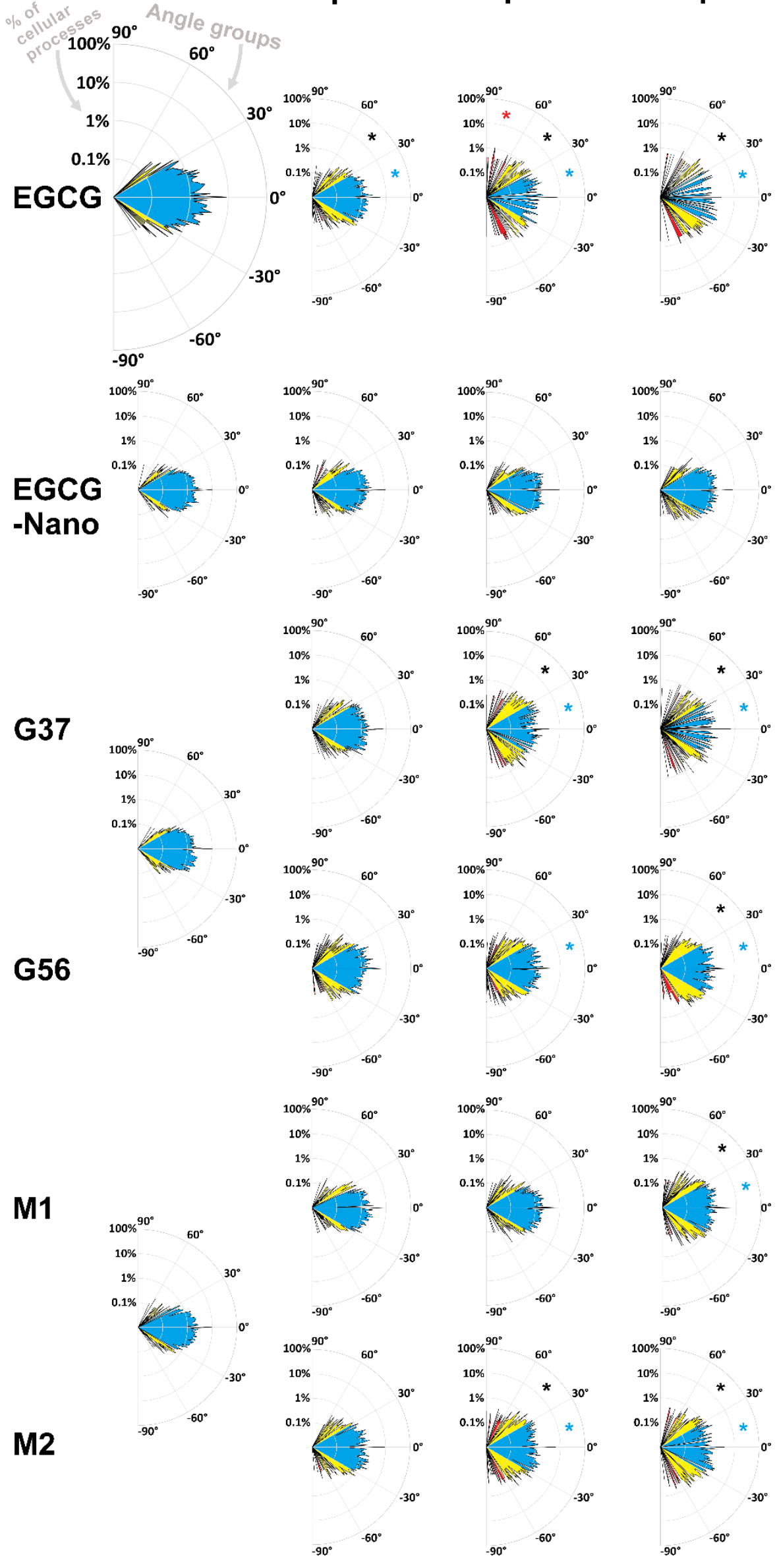


Figure 5. EGCG, Nano-EGCG and structurally related compounds display different effects on the orientation of migrating cells processes. Color-coded polar plot displaying the orientation of cellular processes in relation to the reference axis after exposure of rat neurospheres to increasing concentrations of EGCG, Nano-EGCG, G37, G56, M1 and M2 during $24 \mathrm{~h}$. Blue: angles from -30 to $30^{\circ}$, yellow: angles from $30^{\circ}$ to $60^{\circ}$ and $-30^{\circ}$ to $-60^{\circ}$, red: angles from $60^{\circ}$ to $90^{\circ}$ and $-60^{\circ}$ to $-90^{\circ}$. Results are expressed in percentage of the total angles measured (vertical axis represents percentage in logarithmic scale). Data representing 3 independent experiments, where the angle of 240 cell processes in average per concentration and experiment were measured. ${ }^{*} p<0.05$ versus respective solvent control angle group by two-way ANOVA and Bonferroni analysis (EGCG, G37, G56, M1 and M2 solvent control: 0.01\% DMSO; Nano-EGCG solvent control: $0.1 \%$ Tween ${ }^{\circledR 80)}$. 

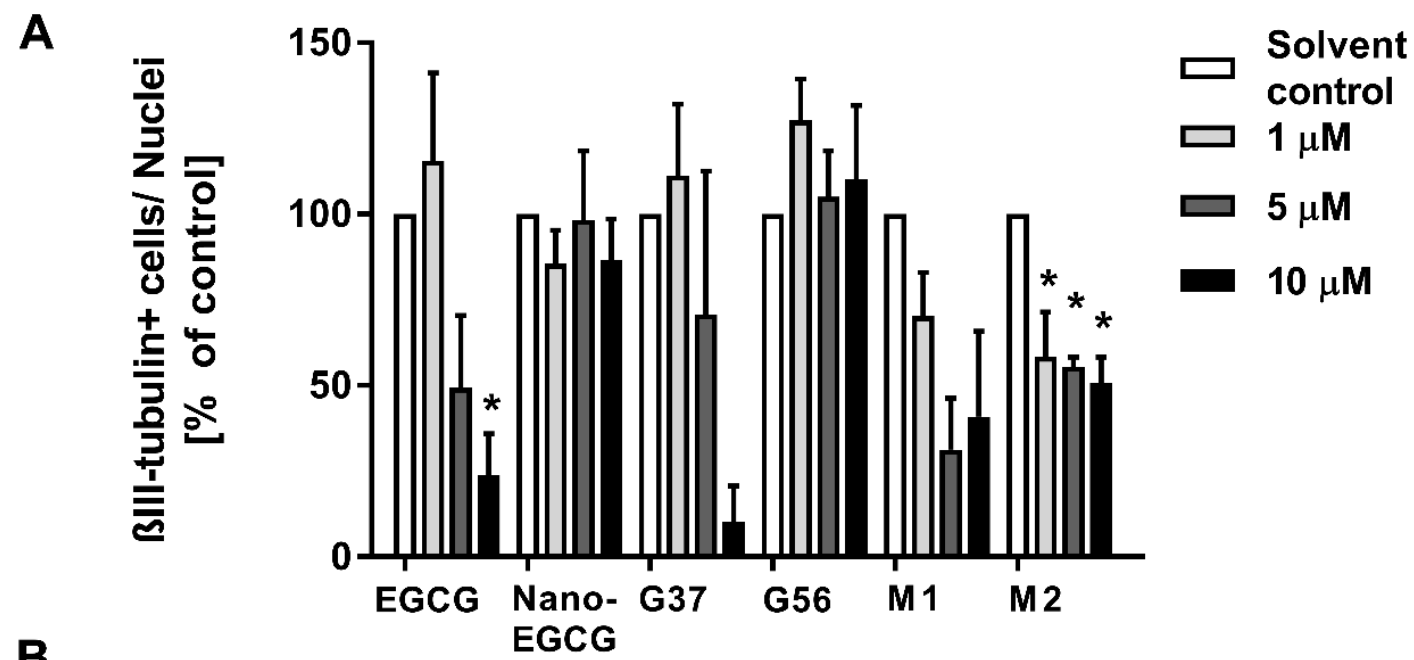

B
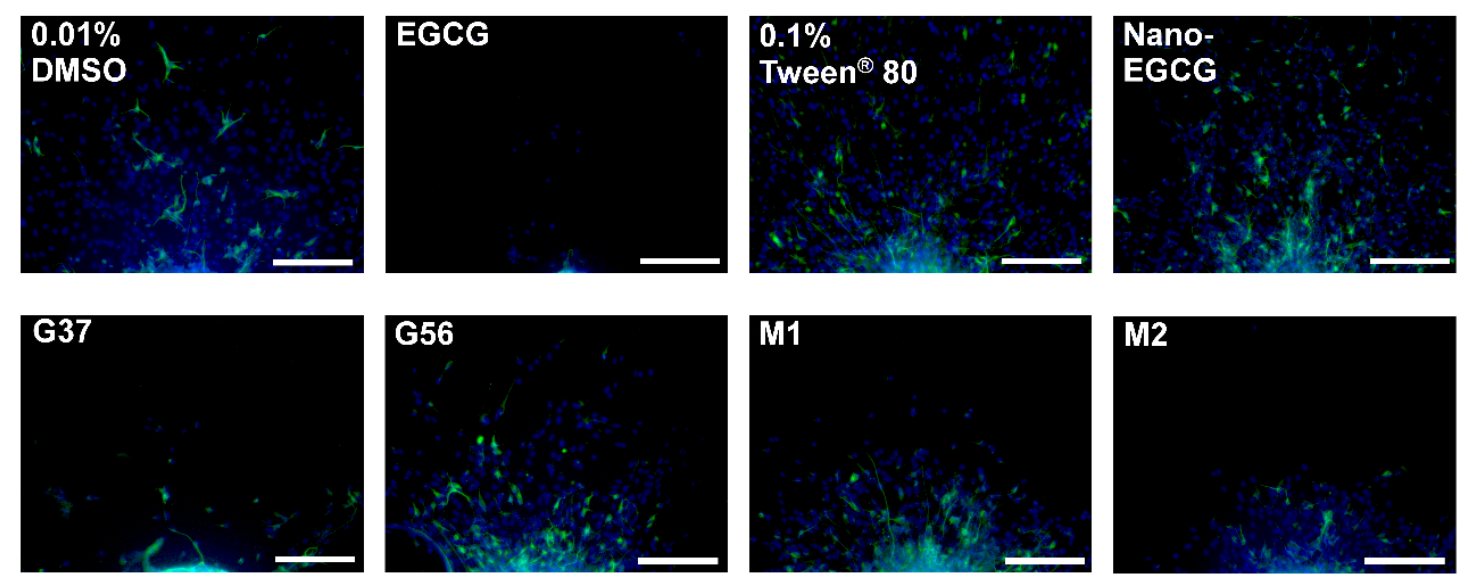

Figure 6. EGCG structurally related compounds display different potencies in disturbing the migration

of young neurons. A. Rat neurospheres were cultured for $24 \mathrm{~h}$ with increasing concentrations of EGCG, Nano-EGCG, G37, G56, M1 and M2. The number of $\beta$ III-tubulin+ cells was manually counted in two areas per neurosphere and five neurospheres per concentration in three independent experiments and divided by the total number of nuclei in the two areas. Results represent the mean \pm SEM in $\%$ of respective solvent control (DMSO $0.01 \%$ for EGCG, G37, G56, M1 and M2 or $0.1 \%$ Tween ${ }^{\circledR} 80$ for Nano-EGCG). ${ }^{*} p<$ 0.05 versus respective solvent control by one-way ANOVA and Bonferroni test. B. Representative pictures of neurospheres exposed to $10 \mu \mathrm{M}$ of the tested compounds and their respective solvent controls. Blue: nuclei staining (Hoechst), green: neuronal marker ( $\beta$ III-tubulin). Scale bars: $100 \mu \mathrm{m}$. 


\section{Supplementary Materials}

A

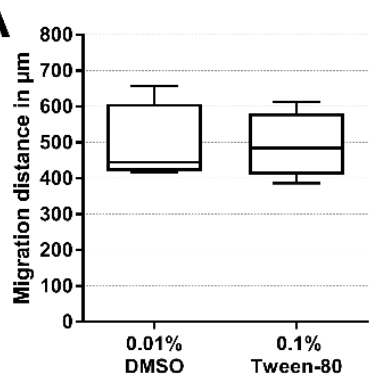

B

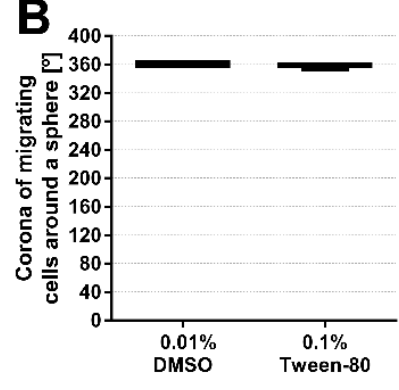

C 垔

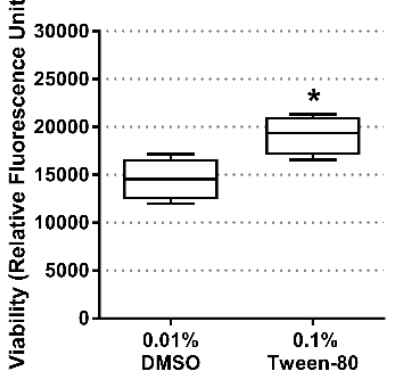

D

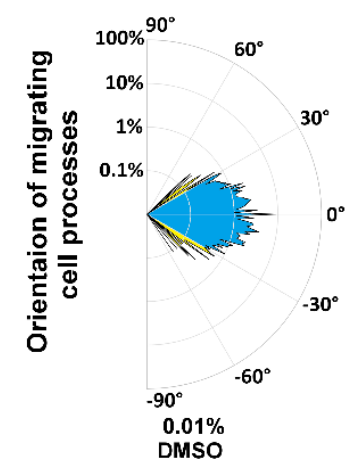

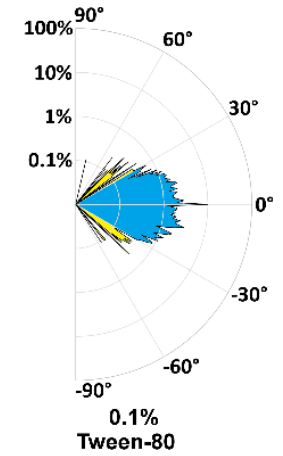

E

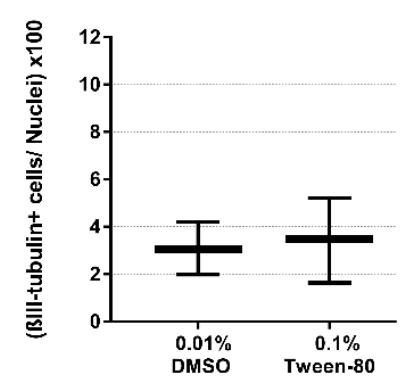

\section{Supplementary Figure 1. Comparison of $0.01 \%$ DMSO and $0.1 \%$ Tween ${ }^{\circledR} 80$ as solvent control substances}

in the rat 'Neurosphere Assay'. Rat neurospheres were cultured for $24 \mathrm{~h}$ with $0.01 \%$ DMSO or $0.1 \%$ Tween ${ }^{\circledR 80}$. All endpoints were evaluated in five neurospheres/concentration in three or four independent experiments. In A, B, C and $E$ results are presented in box-and-whiskers representing minimum and maximum values in. $\mathrm{D}$, color-coded polar plot displaying the orientation of cellular processes in relation to the reference axis. Blue: angles from -30 to $30^{\circ}$, yellow: angles from $30^{\circ}$ to $60^{\circ}$ and $-30^{\circ}$ to $-60^{\circ}$, red: angles from $60^{\circ}$ to $90^{\circ}$ and $-60^{\circ}$ to $-90^{\circ}$. Results are expressed in percentage of the total angles measured (vertical axis represents percentage in logarithmic scale). Data representing 3 independent experiments. ${ }^{*} p<0.05$ versus respective solvent control by Student's-t test. 